\title{
Morphologische Varianz in der Sprache Friedrich Schillers*
}

\author{
Rosemarie Lühr (Jena)
}

\begin{abstract}
The Dictionary of the Complete Vocabulary used by Friedrich Schiller, which is currently being compiled in Jena, displays morphological variants for numerous entries. The declension of foreign expressions in particular exhibit variation and thereby a kind of implicit uncertainty concerning which regularities apply. For instance, three different plural forms of the substantive Zar occur almost next to one another: Czaren, Czars und Czare. Today the speaker would decide on at least one of the variants, but Schiller's fluctuating use of language occurs within the bounds of a single speaker's grammatical competence. It is demonstrated how the influence of dialects, foreign pronounciation and the intended integration of these forms in the High German declension paradigm interact. It becomes obvious that Schiller was "experimenting" with the inflection of foreign terms. The conceptual basis of Optimality Theory makes it especially suitable for the explanation of such idiolect variance. It has the advantage over other theories that a number of competing grammatical systems need not be postulated.
\end{abstract}

\section{$1 \quad$ Vorbemerkung}

Das in Jena entstehende Wörterbuch zum gesamten Wortschatz Friedrich Schillers weist bei zahlreichen Lemmata morphologische Varianten nach. Insbesondere zeigt die Deklination der Fremdwörter Varianz und damit eine implizite Unsicherheit, welcher Regelgebrauch vorliegt. So stehen z.B. bei dem Substantiv Zar drei verschiedene Pluralformen nebeneinander: Czaren, Czars und Czare. Sie finden sich alle im Demetrius, den Schiller mehrfach überarbeitet hat. Zwar vermerkt auch die Duden-Grammatik zur heutigen Deklination der Fremdwörter, dass "nicht selten bei der Pluraldeklination ... Doppelformen auf[treten]", für die "zwei einander widerstrebende Kräfte verantwortlich [sind]", "einerseits ein Beharrungswille, der an den fremden Endungen festhalten möchte, und andererseits ein Streben nach Einheitlichkeit und Ausgleich, das darauf abzielt, auch die Fremdwörter dem deutschen Deklinationssystem anzupassen". Während aber heute ein Sprecher sich zumeist für eine von zwei Varianten entscheidet - er sagt z.B. entweder Balkons oder Balkone, eine Varianz, die dialektal, regional oder sozial bedingt sein kann -, liegt der variierende Sprachgebrauch Schillers in der grammatischen Kompetenz eines einzigen Sprechers. Die den

\footnotetext{
* Einem anonymen Gutachter danke ich für Verbesserungsvorschläge.
} 
Schiller-spezifischen Sprachgebrauch ausmachenden Varianten sind also eine ideolektale Varianz.

Von ihrer Konzeption her bietet sich die Optimalitätstheorie (OT) zur Erklärung auch solcher sprachlicher Varianten an. Denn sie hat gegenüber anderen Theorien den Vorteil, dass für Variation nicht mehrere miteinander konkurrierende Grammatiken vorausgesetzt werden müssen, wobei Variation schlicht besagt, dass statt eines einzigen Kandidaten mehrere Strukturen in einem gegebenen Zeitraum optimal sind. Für die Pluralmorphologie besagt dies, dass oftmals mehrere, darunter fremdsprachliche Varianten miteinander im Wettbewerb stehen, und es ist die Frage, nach welchen Entscheidungskriterien, die auch universeller Natur sein können, also nach welchem Ranking, die eine oder andere Variante gewählt wird. Von den miteinander konkurrierenden Ansätzen zur Beschreibung der Variation innerhalb der OT wird im folgenden der der flexiblen Constrainthierarchie ("Beschränkungshierarchie") aufgegriffen.

Mehrere optimale Outputs ergeben sich aus einem nicht-hierarchischen Verhältnis zwischen Constraints - die grundsätzlich konfligieren - innerhalb eines Rankings. Ein Ranking kann, wenn Constraints nicht hierarchisch gewichtet sind, mehrere optimale Outputs hervorbringen:

Grammatik: C1, C2 _ Output: cand a, cand b (Löhken 1995; 1997: 77).

Nach dem einen Vorschlag ist ein "floating constraint" $X$ in bezug auf Constraint 2 nicht hierarchisch geordnet, er wird aber durch die Constraints 1 und 3 hinsichtlich seiner möglichen Gewichtung eingeschränkt (Reynolds 1994: 113ff.):

a.

\begin{tabular}{|l|l|l|l|l|}
\hline & Constraint 1 & Constraint 2 & Constraint X & Constraint 3 \\
\hline Kandidat A & $* !$ & & & \\
\hline Kandidat B & & $* !$ & & \\
\hline$\rightarrow$ Kandidat C & & & $*$ & \\
\hline
\end{tabular}

b.

\begin{tabular}{|l|l|l|l|l|}
\hline & Constraint 1 & Constraint X & Constraint 2 & Constraint 3 \\
\hline Kandidat A & $* !$ & & & \\
\hline$\rightarrow$ Kandidat B & & & $*$ & \\
\hline Kandidat C & & $* !$ & & \\
\hline
\end{tabular}

In (a) wird der "floating constraint" von Constraint 2 dominiert und Kandidat $\mathrm{C}$ gewinnt, in (b) ist das hierarchische Verhältnis umgekehrt. Dominiert Constraint X, gewinnt Kandidat B. Innerhalb einer undeterminierten Grammatik wird also ein Constraint variabel gewichtet (Zubritskaya 1994; Löhken 1995).

Hinzu kommt ein Lösungsweg, nach dem Variation auf ein nicht-hierarchisches Verhältnis zwischen bestimmten benachbarten Constraints zurückgeht: 
c.

\begin{tabular}{|l|l|l|l|l|}
\hline & Constraint 1 & Constraint 2 & Constraint 3 & Constraint 4 \\
\hline Kandidat A & $* !$ & & & \\
\hline$\rightarrow$ Kandidat B & & $*$ & & \\
\hline$\rightarrow$ Kandidat C & & & $*$ & \\
\hline
\end{tabular}

Dadurch, dass sich Constraint 2 und Constraint 3 im selben Stratum befinden, sind die Verstöße der Kandidaten B und C gleichwertig. Beide Outputs sind gleich und so echte Varianten (nach Löhken 1997: 76ff.). Da (c) die einfachste Lösung darstellt, wird im folgenden danach verfahren.

Wir geben zunächst eine Auswahl aus den bei Schiller belegten Fremdwörtern mit variierender Deklination und ordnen sie Deklinationsklassen zu (B). Dann stellen wir das Ranking zur Zeit Schillers auf, wobei wir die theoretischen Vorgaben von Wunderlichs (1999) OT-Analyse der neuhochdeutschen Pluralformen als Basis nehmen und sie gemäß dem Sprachstand des 18. Jh.s modifizieren (C). Anschließend folgt der Vergleich mit dem heutigen Sprachgebrauch (D).

\section{Die einzelnen Deklinationsklassen}

\section{Klasse der nichtfemininen Substantive auf sonantes $\boldsymbol{r}, \boldsymbol{l}$ (Wunderlich 1999: 3f)}

Unter den eingedeutschten Fremdwörtern gibt es im heutigen Deutsch Nichtfeminina $([+\mathrm{N}]$, [-fem]), deren Plural eine reduzierte Endsilbe aufweist (\{RED(uced) FIN(al) SYLL(able)\}). Diese ist entweder ein Schwa wie in Kollektive oder ein syllabischer Sonorant $(r, l$ oder $n)$ wie in Artikel, Themen, Verben. Die letzte Silbe ist also auf [+son(or)] reduziert und kann nicht betont werden. Es handelt sich um folgende universelle, die Silbenstruktur betreffende Beschränkung: Vokale in unbetonten Silben haben keine phonetische Substanz (Löhken 1997: 282). Im Deutschen ergibt sich dadurch ein Trochäus mit Schwasilbe. Diese prosodische Struktur ist die "kanonische Struktur" nativer Pluralformen (Eisenberg 1991: 48):

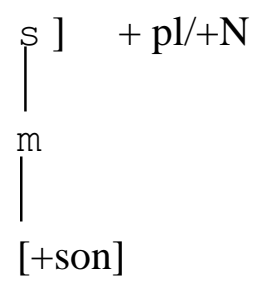

Den ersten Fall repräsentieren bei Schiller Substantive wie Czare oder Succeße, die im Singular auf einen Konsonanten auslauten. Doch da diese Nichtfeminina im Plural häufig Doppelformen nach der starken und schwachen Flexion zeigen, wird für diese Substantive unter III. eine eigene Deklinationsklasse angenommen.

Im zweiten Fall gibt es in der Gegenwartssprache sowohl bei den Feminina als auch bei den Maskulina Plurale, die zusätzlich zu der reduzierten Silbe ein - $n$ aufweisen und im 
Wortauslaut mehrsilbiger Substantive somit gegen *[NO CODA] verstoßen; vgl. die Feminina Muttern, Gabeln und Ziffern und die schwachen Substantive Nachbarn, Ungarn. Wunderlich (1999: 3) setzt hier daher ein Archiphonem an, das nicht weiter spezifiziert zu werden braucht. Während aber Schillers Deklination des Plurals ${ }^{1}$ die Artikel oder die Libertiner dem heutigen Usus entspricht, flektiert er das starke Substantiv Zobel im Plural mit $-n$ :

Nom.Pl.

Libertiner $\{$ Räuber1, Deckbl., NA 3/0 $\}=\{$ Räuber2, Vorsatzbl., NA 3/138\} (heute Pl. Libertiner)

Nom.Pl. Z Zobeln $\quad$ \{Demetr., Koll., NA 11/75\} (heute Pl. Zobel) (Adelung Pl. Zobel)

Dat.Pl.

Zobeln \{Demetr., Koll., NA 11/79\}

\{Demetr., Koll., NA 11/82\}

Nimmt man mit Wunderlich (1999: 13) an, dass \{REDFINSYLL\} die Output-Bedingung für Pluralformen im heutigen Deutschen ist, also Schwa oder sonantisches $r, l$ oder $n$, ist ein zusätzliches Pluralsuffix eigentlich unnötig.

\section{Klasse der schwach flektierenden Substantive (Wunderlich 1999: 6)}

$\mathrm{Zu}$ dieser Klasse gehören Maskulina, die auf -ə enden; z.B. nhd. Hase, Russe. Vgl. die Deklination von Woiwode bei Schiller, dessen Singular in den Formen Nom. Woiwoda, Woiwod und Woiwode (Gen. Woiwoda, Woiwods und Woiwoden, Dat. Woiwod und Woiwoden, Akk. Woiwoden) und dessen Plural in der Form Woiwoden erscheint.

III. Klasse der Nichtfeminina auf Konsonant mit $ə$ - oder $\boldsymbol{n}$-Plural (Wunderlich 1999: 5ff)

Hinzu kommt in der Gegenwartssprache der [e]n-Plural bei den schon erwähnten auf Konsonant auslautenden Maskulina, die in der Regel Menschen als Rollenträger bezeichnen und entsprechend den deutschen Substantiven das Merkmal 'belebt' aufweisen; z.B. Menschen, Student-en, Soldat-en, Doktorand-en, Artist-en, Doktor-en, Pilot-en (Duden Grammatik § 404).

Bei Schiller wird diese Klasse repräsentiert durch:

Nom.Pl.

Ulanen \{WL, Pers.-Verz., NA 8/7\} (Sg. Ulan)

$\{$ WL, 1.A. [vor 1], NA 8/9\}

Nom.Pl. $\quad$ Czaren $\quad$ Demetr., Koll., NA 11/64\} (heute Pl. Zaren).

Doch kommt neben Czaren auch die genannte Form Czare vor.

Nom.Pl.

Czare \{Demetr., Koll., NA 11/78\}

\{Demetr., Koll., NA 11/80\}

Auch Tierbezeichnungen sind bei Schiller teils mit schwankender Deklination belegt:

Dat.P1.

Skorpionen \{Macbeth, III/5, NA 13/118\} (heute Pl. Skorpione)

(Adelung Pl. Skorpione)

\footnotetext{
${ }^{1}$ Sämtliche Schiller-Belege sind dem Morphologie-Teil des oben genannten "Wörterbuchs der Sprache Friedrich Schillers" entnommen. Zugrunde liegt die "Nationalausgabe" von Schillers Werken.
} 
Akk.Pl. Skorpionen $\{$ KuL V/3, NA 5/93\}

Nom.Pl. Leoparde $\{$ Räuber1, I/2, NA 3/31 $\}=\{$ Räuber2, I/7, NA 3/158 $\}$ (heute Pl. Leoparden)

Akk.Pl. Leoparden $\{$ Handsch., [35], NA 1/367 $\}=\{$ Handschuh, [35], NA 2-I/275 $\}$

Ein [e]n-Plural erscheint neben einem ə-Plural weiterhin bei unbelebten Nichtfeminina, die im Neuhochdeutschen nur einen Plural auf - 2 haben, wie etwa das Maskulinum Aspecten/Aspekten mit [e]n-Plural zum Singular Aspekt; gegenüber Succeße nur mit ə-Plural:

Nom.Pl. $\quad$ Succeße $\quad$ SDemetr., Studienh., NA 11/95\} (heute Pl. Suksesse)

\{Demetr., Studienh., NA 11/121\}

\{Demetr., Skizz., NA 11/158\}

\{Warb., NA 12/185\}

Dat.Pl. Succeßen \{Demetr., Szen., NA 11/212\}

Successen $\{$ An Goethe, 22.7.1800, NA 30/174\}

Akk.Pl. $\quad$ Successe \{Demetr., Entw., NA 11/273\}

Häufig hat man aber auch bei den unbelebten Substantiven ein Schwanken zwischen beiden Pluralformen beim selben Wort:

Nom.Pl.

Zinsen \{Phil.Briefe, Aufopf., NA 20/123\} (heute Pl. Zinsen neben Zinse) (Adelung Pl. Zinse)

\{An H.v.Wolzogen, 10.7.1788, NA 25/79\}

Zinse $\quad$ An Chr.Schiller, 6.11.1782, NA 23/50\}

Dat.Pl. Zinsen $\quad$ \{30j.Kr., 3.B., NA 18/239\}

\{An Huber, 28.2.1785, NA 23/181\}

\{An Ch.v.Lengefeld, 29.10.1789, NA 25/309\}

Zinßen \{An J.G.Franckh 23.5.1802, NA 31/136\}

Akk.Pl.

Zinsen \{Melanch., 46, NA 1/113\}

\{Die schl.Mon., 99, NA 1/127\}

\{Räuber2, I/3, NA 3/150\}

$\{$ DK1, II/2 [1347], NA 6/70 $\}=\{$ DKTh.-Frg.1786, II/3 [1595], NA

$6 / 418\}=\{\mathrm{DK} 4, \mathrm{II} / 2$ [1157], NA 7/416 $\}$

\{Geisters.F.an O.,9.Br., NA 16/153\}

\{Gesetzgebung, Solon, NA 17/430\}

\{30j.Kr., 4.B., NA 18/285\}

\{An Körner, 18.-19.8.1787, NA 24/137\} 2x

\{An W.v.Wolzogen, 1.9.1788, NA 25/103\}

Nom.Pl.

Thronen $\{$ Sonne, [35], NA 1/52\} (heute Pl. Throne) (Adelung Pl. Throne)

Throne \{Melanch., [31], NA 1/113\}

\{DK3, I/1, NA 7/237\}

\{Abf.d.Niederl., 1.B., NA 17/70\}

\{2.Rede, NA 20/36\}

\{Anm. u. Würde, NA 20/300\}

Trone $\{$ DKTh.-Frg.1785, I/1 [320], NA 6/360\}

\{Reisender Däne, NA 20/106\}

Gen.Pl. Throne $\{$ DKTh.-Frg.1785, I/1 [106], NA 6/351\}

\{Abf.d.Niederl., 2.B., NA 17/128\} 


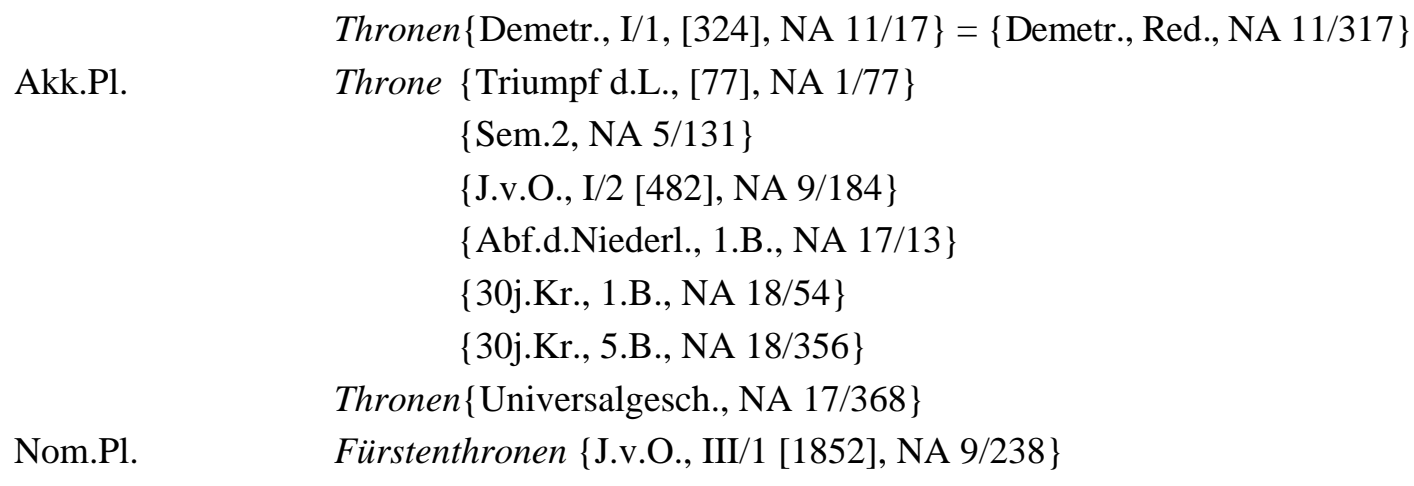

Gleiches gilt für suffigierte Neutra, die im Neuhochdeutschen einen regelhaften Plural auf -ə haben:

Nom.Pl. Citaten $\quad$ An Crusius, 24.1.1788, NA 25/11\} 2x (heute Pl. Zitate)

Citate $\{$ An Cotta, 30.1.1795, NA 27/134\}

Vgl. demgegenüber nhd. Aggregate, Attentate.

Auch sonst begegnet bei Neutra ein Schwanken zwischen - $e$ und -[e]n-Formen:

Nom.Sg. Atom \{Phil. Briefe, Liebe, NA 20/122\} (heute Pl. Atome) (Adelung Pl. Atome)

Atome $\{$ Spaz.Linden, NA 22/75\}

\{Spaz.Linden, NA 22/75\}

\{Br.D.K.12, NA 22/174\}

Dat.Sg. Atomen $\{$ Spaz.Linden, NA 22/78\}

Akk.Pl. Atomen $\{$ Freundsch., [23], NA 1/110\}

\{Rhein.Thal., Ank., NA 22/96\}

Atome \{J.v.O., III/6 [2347], NA 9/258\}

Nom.Pl. Symbole $\quad$ Fragm.ästh.Vorl., NA 21/82\} (heute Pl. Symbole)

Gen.Pl. Symbolen $\{$ Moses, NA 17/382\}

Symbole \{Her.Prop., NA 22/306\}

Dat.Pl. Symbolen $\quad$ Zerstr. Betr., NA 20/237\}

\{ Ästh. Erz., 9.Brief, NA 20/336\}

Akk.Pl. Symbolen $\{$ Stehende Schaubühne, NA 20/99\}

Symbole \{An Goethe, 17.8.1795, NA 28/28\}

\{An Goethe, 28.11.1797, NA 29/162\}

Nom.Pl. Symptome $\quad$ Pathet., NA 20/200\} (heute Pl. Symptome)

Dat.Pl. Symptomen \{Malth., NA 12/30\}

\{Teut.Aen., NA 22/186\}

\{An Körner, 21.5.1791, NA 26/84\}

Akk.Pl. Symptomen $\quad$ Malth., NA 12/47\}

Symptome Malth., NA 12/77\}

\{Geisters.1.B., NA 16/98\}

Nom.Pl. Pasquillen $\quad$ Abf.d.Niederl., 2.B., NA 17/146\} (heute das Pasquill 'anonyme Schmähschrift'

Akk.Pl. $\quad$ Pasquille $\{$ Abf.d.Niederl., 3.B., NA 17/186\}

\{Egmont, NA 17/296\}

\{An Körner, 23.2.1788, NA 25/19\} 
D.h., der $e[n]$-Plural ist im älteren Deutsch bei den Nichtfeminina mit einem auf Konsonant auslautenden Singular noch nicht auf die Klasse [+ belebt] festgelegt. Auch ist in dieser Klasse ein Schwanken zwischen ə- und $n$-Plural möglich. Daher ist wohl anzunehmen, dass in der Sprache Schillers nicht nur sonantes $l$ wie in Zobeln mit dem Plural- $n$ versehen werden konnte, sondern auch die Reduktionsform ə wie in Czaren neben Czare.

\section{Klasse der umgelauteten Substantive (Wunderlich 1999: 4f)}

Unter den Nichtfeminina mit Umlaut, Klasse IV, fällt die Pluralbildung von Vogt auf. Während es im Neuhochdeutschen Vögte heißt, kommt bei Schiller neben Vögte einmal umlautloses Vogte vor - im Grimmschen Wörterbuch ist diese Form nicht verzeichnet.

\{Tell, I/2, [283ff.], NA 10/144\}

Es kommt kein Fischerkahn zu uns herüber,

Der nicht ein neues Unheil und Gewalt-

Beginnen von den Vogten uns verkündet.

$\begin{array}{lll}\text { Nom.Pl. } & \text { Vögte } & \{\text { Tell, II/2, [1424], NA 10/191\} (heute Pl. Vögte) (Adelung Pl. Vögte) } \\ \text { Gen.Pl. } & \text { Vögte } & \{\text { Tell, I/2, [191], NA 10/141\} } \\ & & \{\text { Tell, II/2, [1050], NA 10/176\} } \\ & \{\text { Tell, II/2, [1325], NA 10/187\} } \\ \text { Dat.Pl. } & \text { Vogten } & \{\text { Tell, I/2, [285], NA 10/144\} } \\ \text { Akk.Pl. } & \text { Vögte } & \{\text { Tell, II/2, [1367], NA 10/188\} } \\ & & \{\text { Tell, III/1, [1518], NA 10/195\} } \\ & \{\text { Moses, NA 17/378 } \\ & \{\text { An Ch.v.Lengefeld, 5.2.1789, NA 25/199 }\end{array}$

Da Schiller Vogt im Plural einmal wie Czare, einmal wie Gäste flektiert, ist also das Merkmal $[$ COR $($ onal $)=$ Umlaut $]$ oder [+front] bei diesem Stamm (Wiese 1996) nicht fest. Grundsätzlich handelt es sich dabei um eine Treuebeschränkung des Typs $\{$ MAX(FEATURE)\}, die das Erscheinen von Merkmalen in einer abgeleiteten Struktur fordert (Wunderlich 1999: 4). Doch kommen bei Schiller auch in einheimischen Wörtern umlautlose und umlauthaltige Pluralformen vor, bei denen die heutige Hochsprache nur eine Form hat; vgl. etwa Tage neben Täge $e^{2}$, wo ebensowenig wie bei Vogte und Vögte ein Bedeutungsunterschied herrscht. Der Fall liegt anders als etwa bei Bande 'Freundschaftsbande' und Bände 'Buchbände'.

\section{Klasse mit unterschiedlichen Singular- und Pluralstämmen (Wunderlich 1999: 6)}

Ein Bespiel für diese Klasse ist der Plural:

Nom.Pl. Vesicatorien $\quad$ An Körner, 22.2.1791, NA 26/75\}

\{An Goethe, 4.11.1799, NA 30/115\}

Zu Vesikatorium 'medizinisches Zugmittel'.

\footnotetext{
${ }^{2}$ In den heutigen süddeutschen Varietäten wird statt des ə-Plurals und bei $e$-Apokope kompensatorisch der Umlautplural verwendet (Wegener 2001: 270).
} 
VI. Klasse mit Plural auf (nichtfranzösischem) -s (Wunderlich 1999: 2f.)

Betrachtet man nun den s-Plural von Zars

$\begin{array}{ccl}\text { Nom.Pl. } & \text { Czars } & \{\text { Demetr., Koll., NA 11/76\} } \\ & & \{\text { Demetr., Koll., NA 11/76\} } \\ \text { Akk.Pl. } & \text { Czars } & \{\text { Demetr., Koll., NA 11/73 }\end{array}$

so hat Schiller offensichtlich den Plural an den Typ Thans, einem Lehnwort aus engl. Thanes 'angelsächsische Hofbeamte' (vom 9. bis 15. Jh.), angepaßt:

Nom.Pl.

Thans $\quad$ Macbeth, I/8, NA 13/87\}

\{Macbeth, III/8, NA 13/125\}

\{Macbeth, V/3, NA 13/151\}

\{Macbeth, V/4, NA 13/153\}

VII. Klasse mit Plural auf -er (Wunderlich 1999: 5)

Nach der Duden Grammatik (§ 405) haben nur vier Fremdwörter einen Plural auf -er: Hospitäler, Regiment-er, Ski-er, Spitäl-er.

Schiller bietet aber:

Nom.Pl.

Veteraner \{Abf.d.Niederl., 3.B., NA 17/267\}

Akk.Pl.

Billetter $\quad\{\mathrm{KuL} \mathrm{I} / 1, \mathrm{NA} 5 / 6\}$

Im Grimmschen Wörterbuch (XII II, 22) wird als ungewöhnlicher Plural für K.Ph.Moritz, Anton Reiser 34, die Form Veteraner gebucht. Sie bezieht sich dort auf Schüler (die vier öbersten (schüler), welche er auch im scherz veteraner hiesz) und ist sicher eine Reimbildung auf dieses Wort. Für Schiller bietet sich aber das Vorbild Krieger an, da man als Eindeutschung von Veteran das deutsche Wort Altkrieger erwogen hat. Veteraner ist jedenfalls ein Sonderfall, der nicht der regulären Pluralbildung folgt. Gleiches gilt für den Plural Billetter, den Schiller in "Kabale und Liebe" der schwatzhaften Mutter der Luise in den Mund legt ${ }^{3}$.

VIII. Klasse der Feminina auf -n (Wunderlich 1999: 5)

Die Deklination der fremdstämmigen Feminina mit deutschen Endungen ist unproblematisch; sie bilden regelmäßig den [e]n-Plural; vgl. etwa Turnüre 'Gewandtheit, List', Taxe 'Steuer, Gebühr', Tour 'Drehung, Ausflug, Rundfahrt', Trakasserie 'Schikane', Vignette 'ornamentale Verzierung in einem Buch', Zechine 'italienische (ursprünglich venezianische) Münzeinheit', Zession 'Abtretung', Interzession 'Intervention', Konzession 'Zugeständnis', Sukzession 'Nachfolge, kontinuierliche (zeitliche) Abfolge', Zelebrität 'Berühmtheit', Zeremonie 'feierliche Handlung':

\footnotetext{
${ }^{3}$ Im 18. Jh. war der er-Plural produktiv, sowohl in einheimischen Wörtern als auch in Fremdwörtern. Im Alemannischen, Bairischen, Fränkischen, Hessischen, Pfälzischen, Schwäbischen hat sich dieser Plural oft bis heute erhalten (Wegener 2001: 267).
} 
Dat.P1.

Dat.Pl.

Dat.Pl.

Akk.Pl.

Gen.Pl.

Dat.Pl.

Akk.Pl.

Nom.Pl.

Dat.Pl.

Akk.Pl.

Akk.Pl.

Gen.Pl.

Dat.Pl.

Akk.Pl.

Gen.Pl.

Akk.Pl.

Dat.Pl.

Nom.Pl.

Dat.Pl.

Akk.Pl.

Dat.Pl.

Dat.Pl.

Nom.Pl.(?)
Tournüren \{An Ch.Schiller, 13?.8.1802, NA 31/156 \}

Taxen \{30j.Kr., 3.B., NA 18/241\}

\{30j.Kr., 5.B., NA 18/365\}

Touren \{An Körner, 23.2.1793, NA 26/216 \}

Tracasserien \{An Cotta, 6.2.1801, NA 31/7 \}

Vignetten \{An Göschen, 3.7.1791, NA 26/92 \}

\{An Crusius, 5.10.1792, NA 26/156 \}

Vignetten \{An Goethe, 21.5.1795, NA 27/188\}

Vignetten \{An Crusius, 19.4.1789, NA 25/247\}

\{An Cotta, 1.2.1797, NA 29/42\}

\{An Crusius, 3.4.1803, NA 32/29\}

Zecchinen $\{$ Fiesko I, I/9, NA 4/27\} = Zechinen $\{$ Fiesko II, I/9, NA 4/144 \}

$\{$ Fiesko I, I/9, NA 4/27\} = Zechinen $\{$ Fiesko II, I/9, NA 4/144\}

Zecchinen $\{$ Fiesko I, II/4, NA 4/43\} = Zechinen $\{$ Fiesko II, II/1, NA 4/149\}

Zecchinen $\{$ Fiesko I, I/2, NA 4/16 \} = Zechinen $\{$ Fiesko II, I/2, NA 4/133\}

\{Fiesko I, I/9, NA 4/29\} = Zechinen $\{$ Fiesko II, I/9, NA 4/146

\{Fiesko I, III/4, NA 4/71 $\}=$ Zechinen $\{$ Fiesko II, III/3, NA 4/184

\{An Goethe, 8.12.1795, NA 28/127\}

Cessionen $\{30$ j.Kr., 5.B., NA 18/335\}

Interzessionen \{Egmont, NA 17/307\}

Intercessionen \{Egmont, NA 17/306 \}

Concessionen \{Egmont, NA 17/298\}

Succeßionen $\quad$ PPhil. Briefe, Idee, NA 20/119\}

Succeßionen \{Vers.Zus. § 2, NA 20/42 \}

Celebritäten \{Ber.Frau, [102], NA 1/199\}

Ceremonien $\quad$ Moses, NA 17/384\}

Ceremonien $\quad$ Moses, NA 17/388\}

\{An Körner, 18.-19.8.1787, NA 24/137\}

Ceremonien $\quad$ DDemetr., Studienh., NA 11/109\}

\{An Voigt, 25?.4.1789, NA 25/249\}

\{An Körner, 30.4.1789, NA 25/252\}

Einweyhungs Ceremonien \{Moses, NA 17/395\}

Leichenzeremonien \{Anm. u. Würde, NA 20/308 Anm.

Schinders Ceremonien $\quad$ Räuber1, II/3, NA 3/62

IX. Klasse mit fremdstämmigen Endungen (Wunderlich 1999: 9)

In vielen Fällen wird gegenüber den Fremdwörtern mit einheimischen Pluralendungen aber die fremdsprachliche Pluralendung beibehalten; dabei handelt es sich zumeist um die französische Pluralendung ${ }^{4}$.

Im Maskulinum:

Nom.Pl.

Abbes $\{$ Pol., NA 12/97\} (heute Pl. Abbés)

\footnotetext{
${ }^{4}$ Zum Eindringen des $s$-Plurals aus dem Französischen im 18. Jh. vgl. Bornschein/Butt 1987.
} 
Im Neutrum:

Akk.Pl. Lavements \{An Stark, 24?.5.1791, NA 26/89\}

(frz. lavement 'Klistier; Darmeinlauf, -spülung [meist mit warmem Wasser]')

Im Femininum:

Dat.Pl. Longeurs $\quad$ An Goethe, 25.6.1798, NA 29/243\}

(frz. longueur 'Länge, Weitschweifigkeit')

Akk.Pl. Longeurs \{An Ch.v.Schiller, 6.7.1803, NA 32/48\}

Akk.Pl. Saisons $\quad$ An Körner, 15?.4.1788, NA 25/40\}

Dat.Pl. Vauxhalls $\quad$ An Zumsteeg, 19.1.1784, NA 23/130\} (frz. vauxhall 'Tanzveranstaltung')

Bei Neutra kommt auch die lateinische Pluralendung vor:

Nom.Pl. $\quad$ Lumina \{W.T., I/1 [22], NA 8/178\} (lat. lumen 'leuchtender Himmelskörper')

Akk.Pl. $\quad$ Lustra $\{$ Xen.186, NA 1/332\} (lat. lustrum 'Zeitraum von fünf Jahren')

\section{Mischklasse ${ }^{5}$}

Oftmals erscheint aber die fremdsprachliche Endung neben der einheimischen:

Vgl. das Maskulinum:

Nom.Pl. Libertiner $\{$ Räuber1, Deckbl., NA 3/0 $\}=\{$ Räuber2, Vorsatzbl., NA 3/138 $\}$ (heute Libertiner 'Freigeist, Freidenker; leichtsinniger, zügelloser Mensch' Libertins \{Pol., NA 12/99\}

Das Neutrum:

Nom.Pl.

Meubles \{Paras., V/3, NA 15-II/161\}

\{An Körner, 8.12.1787, NA 24/181\}

Dat.Pl.

Meuebles \{An Chr. Reinwald, 1.8.1787, NA 24/120\}

Moeubles \{An Körner, 28.-31.7.1787, NA 24/114\}

Meubles $\{$ An Körner, 1.2.1796, NA 28/178\}

\{An Körner, 20.7.1801, NA 31/53\}

Akk.Pl.

Meublen \{Geisters.1.B., NA 16/59\}

\{Gesetzgebung, NA 17/417\}

\{An Hufeland, 21.3.1789, NA 25/229\}

Meubles \{Schwäb.MA, NA 22/187\}

\{An H.v.Wolzogen, 28.7.1783, NA 23/102 \}

\{An Ch.v.Lengefeld, 11.4.1788, NA 25/39\}

\{An Körner, 10.3.1789, NA 25/227\}

\{An Körner, 13.5.1789, NA 25/254\}

\{An Körner, 24.12.1789, NA 25/374\}

\{An Körner, 27.12.1789, NA 25/376\}

\{An L.v.Lengefeld, 9.1.1790, NA 25/385\} 2x

\{An C.v.Beulwitz/Ch.v.Lengefeld, 25.1.1790, NA 25/400\}

\{An Körner, 29.2.1796, NA 28/196\}

\{An Goethe, 24.12.1798, NA 30/19\}

\footnotetext{
${ }^{5}$ Bei Wunderlich fehlen für diesen Typ Beispiele.
} 
moeubles \{An Körner, 6.9.1785, NA 24/17\}

Vgl. die Feminina:

Nom.Pl.

Adresses \{An Körner, 12.2.1788, NA 25/15\} (heute Pl. Adressen)

Dat.Pl.

Adressen \{An Göpferdt, 11?.12.1796, NA 29/25\}

Akk.Pl.

Addressen \{An Chr.F.Jacobi, 6.11.1782, NA 23/46\}

\{An Chr.Schiller, 6.11.1782, NA 23/49\}

\{An Streicher, 14.1.1783, NA 23/63\}

\{An H.v.Wolzogen, 1.2.1783, NA 23/64\}

\{An H.v.Wolzogen, 27.3.1783, NA 23/73\}

Adressen \{An W.v.Wolzogen, 8.3.1790, NA 26/5 \}

Nom.Pl.

Reflexionen \{Iph.i.A., Anm., NA 15-I/78\} (Adelung Pl. Reflexionen)

\{Iph.i.A., Anm., NA 15-I/79\}

\{An Huber, 2.1.1789, NA 25/170\}

\{An Goethe, 6.3.1798, NA 29/215\}

Gen.Pl. Reflexionen $\quad$ An Körner, 6.10.1787, NA 24/161\}

Akk.Pl. Reflexions $\quad$ An C.v.Beulwitz/Ch.v.Lengefeld, 4.12.1788, NA 25/153\}

Reflexionen \{An Goethe, 20.7.1798, NA 29/256 \}

\{An Goethe, 4.12.1798, NA 30/9\} 2x

Wie die Auswertung des Morphologieteils des Schiller-Wörterbuchs (vgl. Anm. 1) zeigt, finden sich die französischen Endungen dabei überwiegend in Schillers Briefen. Konkurrieren diese Endungen mit einheimischen, handelt es sich in der Regel um frz. $-s$ vs. dt. $-[e] n$.

Die aus dem Schiller-Wortschatz ausgewählten Fremdwörter geben nun folgende Deklinationsklassen:

\section{Klasse der nichtfemininen Substantive auf sonantisches $r, l$}

Libertiner, Zobeln

\section{Klasse der schwach flektierenden Substantive}

Woiwoden

\section{Klasse der Nichtfeminina auf Konsonant mit ə- oder $\boldsymbol{n}$-Plural}

Ulanen,

Czare/Czaren

Skorpionen

Leoparde/Leoparden

Aspekten

Suczesse

Zinse/Zinsen

Throne/Thronen

Citate/Citaten

Atome/Atomen 
Symbole/Symbolen

Symptome/Symptomen

Pasquille/Pasquillen

IV. Klasse der umgelauteten Substantive

Vögte/Vogte

\section{Klasse mit unterschiedlichen Singular- und Pluralstämmen}

Vesicatorien zu Vesikatorium

\section{Klasse mit Plural auf (nichtfranzösischem) -s}

Czars, Thans

VII. Klasse mit Plural auf -er

Billetter, Veteraner

\section{Klasse der Feminina auf $-n$}

Tournüren, Taxen, Touren, Tracasserien, Vignetten, Zecchinen, Cessionen, Interzessionen, Concessionen, Succeßionen, Celebritäten, Ceremonien, Einweyhungs Ceremonien, Leichenzeremonien, Schinders Ceremonien

\section{Klasse mit fremdstämmigen Endungen}

Abbes, Lavements, Longeurs, Saisons, Vauxhalls

Lumina, Lustra

\section{Mischklasse}

Libertiner/Libertins

Meublen/Meubles

Adressen/Adresses

Reflexionen/Reflexions

\section{Klasse mit Plural auf $-a$}

Lustra

\section{$3 \quad$ Ranking}

Vor dem Ranking der Constraints sind nun Besonderheiten und Regularitäten in der Pluralbildung der Fremdwörter bei Schiller anzugeben. Ein Pluralmorphem, das zweifellos eine Sonderstellung einnimmt, ist der $s$-Plural: Er kommt in dem englischen Fremdwort Than vor und danach in Czar, weiterhin in den direkten Übernahmen aus dem Französischen, 
wobei $s$ hier wohl nicht artikuliert ist. In Fremdwörtern ist das Plural-s also lexikonbasiert. In einheimischen Wörtern zeigt der Plural Uhus neben Uhue dagegen, dass bei Schiller auch ein auslautender voller Vokal einen solchen Plural bedingt.

Nom.Pl.

Uhus \{Räuber1, IV/5, NA 3/112\}

Uhue \{Räuber2, IV/17, NA 3/214\}

Wegen seines vollen Auslautsvokals gehört $U h u$ dabei zu den "untypischen" Substantiven des Deutschen.

Lexemgebunden ist der $a$-Plural bei lateinischen Fremdwörtern sowie der $n$-Plural bei Substantiven mit verschiedenen Singular- und Pluralstämmen; vgl. dazu nhd. Virus, Plural Viren.

Auch dem Pluralzeichen -er kommt im Fremdwortschatz Schillers Sonderstatus zu, sofern Veteraner nach Krieger gebildet und Billetter eine Kunstbildung ist. Weiterhin kann ein lexikalisches Umlaut-Merkmal mit dem Plural verbunden sein: Voge/Vögte.

Damit ergeben sich folgende Regeln für die Deklination der Fremdwörter:

Maskulina auf - ə flektieren schwach und haben demnach $n$-Plural.

a. Feminina haben $n$-Plural.

b. Nichtfeminina auf auslautendem Konsonant haben $ə$ - oder $n$-Plural.

c. Nichtfeminina auf sonantischem $l, r$ haben einen unmarkierten oder einen $n$-Plural.

d. Offensichtliche Fremdwörter haben $s$-Plural.

e. Ein lexikalisches Umlaut-Merkmal kann im Plural vorhanden sein oder fehlen.

f. Der $e r$-Plural ist lexemgebunden.

g. Fremdwörter aus dem Lateinischen haben $a$ - oder - bei unterschiedlichem Stamm - $n$-Plural.

Was nun das Ranking selbst angeht, so wird mit Wunderlich (1999: 13) davon ausgegangen, dass der Input aus einem Lexikoneintrag zusammen mit dem Merkmal [+Pl] besteht. Wenn das Lexikon schon eine Pluralform anbietet, wird diese Form gewählt. ${ }^{6}$ Das sind für unsere Auswahl an Fremdwörtern die Formen nach den Punkten f) und g), also der er-Plural, der lateinische $a$-Plural und der $n$-Plural bei unterschiedlichen Singular- und Pluralstämmen. Wenn jedoch der Lexikoneintrag keine spezielle Pluralform enthält, muss die richtige Form aus der Anzahl der Kandidaten gewählt werden. Diese Kandidatenmenge besteht aus der Input-Form, einer Form mit ə, Formen mit $n$ - oder $s$-Suffix und einem Merkmal [COR], sofern der Lexikoneintrag dieses Merkmal in der abgeleiteten Struktur erfordert. Weiterhin gilt, dass die Kandidaten, die mit einem Suffix gebildet werden, die Bedingungen für dessen Auftreten erfüllen müssen. Z.B. erscheint das Suffix $n$ obligatorisch nur bei Maskulina, die auf $ə$ enden, und bei Feminina. Dagegen kommt in diesen Fällen kein $s$-Suffix vor. Man könnte für die Suffixe so einen allgemeinen Constraint \{EXPONENCE\} annehmen, der besagt, dass jede Wortform eines Paradigmas den jeweils relevanten morphologischen Marker

${ }^{6}$ Zu Pluralformen, die das Lexikon bereitstellt, vgl. auch Wegener 2001: 287. 
aufweist (Raffelsiefen 1995: 28). Wie Löhken (1997: 72) ausführt, ist dieser Constraint dann je nach Art der Markierung einzelsprachlich weiter zu spezifizieren.

Da nun mit Ausnahme der Fälle a) und b), die die schwachen Maskulina auf -ə und die Feminina betreffen, alle anderen Regeln Varianten zulassen, selbst das im Neuhochdeutschen feste Merkmal [COR], haben die Beschränkungen \{MAX(COR), REDFINSYLL, N-PLUR, S-PLUR \} ein gleiches Ranking. Dies gilt auch für den $s$-Plural, denn dieser hat bei den Fremdwörtern oftmals eine Variante auf $-n$ oder -ə neben sich. Hinzu kommen zwei weitere Constraints, einerseits ein die Verschiedenheit von Singular und Plural fordernder Constraint \{MARKPLUR \} und andererseits ein Constraint $\{$ NO AFFIX\}, der jede Affigierung verbietet, Constraints, die neben \{REDFINSYLL\} stehen. Zu beachten ist dabei, dass - $ə$ nicht als Pluralaffix zählt, sondern sich durch \{REDFINSYLL\} ergibt. Da jedoch ein -ə im Plural eine Veränderung des Outputs gegenüber dem Input, dem Singular, verursacht, wirkt hier ein Constraint $\{$ DEP(endence)(Mora) $\}$ : Moren im Output müssen eine Entsprechung im Input haben.

a.

\begin{tabular}{|c|c|c|c|c|c|c|c|c|c|c|}
\hline & $\begin{array}{l}\text { ER- } \\
\text { PL }\end{array}$ & $\begin{array}{l}\text { A- } \\
\text { PL }\end{array}$ & $\begin{array}{l}\text { N-PL } \\
\text { STAMM- } \\
\text { DIFF }\end{array}$ & $\begin{array}{l}\text { MAX } \\
\text { (Cor) }\end{array}$ & $\begin{array}{l}\text { REDFIN } \\
\text { SYLL }\end{array}$ & $\begin{array}{l}\mathrm{N}- \\
\mathrm{Pl}\end{array}$ & $\begin{array}{l}\text { S- } \\
\text { PL }\end{array}$ & $\begin{array}{l}\text { MARK } \\
\text { PL }\end{array}$ & $\begin{array}{l}\text { DEP } \\
\text { (Mora) }\end{array}$ & $\begin{array}{l}\text { NO } \\
\text { Affix }\end{array}$ \\
\hline Veteraner & $\sqrt{ }$ & & & & & & & & $*$ & $*$ \\
\hline Lustra & & $\sqrt{ }$ & & & $*$ & & & & $*$ & $*$ \\
\hline Vesicatorien & & & $\sqrt{ }$ & & & & & & $*$ & $*$ \\
\hline Libertiner & & & & & & $*$ & $*$ & $*$ & & \\
\hline Zobeln & & & & & $*$ & & $*$ & & & $*$ \\
\hline Woiwoden & & & & & & & $*$ & & & $*$ \\
\hline Czare & & & & & & $*$ & $*$ & & $*$ & \\
\hline Czaren & & & & & & & $*$ & & $*$ & $*$ \\
\hline Czars & & & & & $*$ & $*$ & 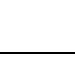 & & & $*$ \\
\hline Vogte & & & & & & $*$ & $*$ & & $*$ & \\
\hline Vögte & & & & & & $*$ & $*$ & & $*$ & \\
\hline Taxen & & & & & & & $*$ & & & $*$ \\
\hline Saisons & & & & & $*$ & $*$ & & & & $*$ \\
\hline Meubles & & & & & $*$ & $*$ & & & & $*$ \\
\hline Meublen & & & & & $*$ & & $*$ & & & $*$ \\
\hline
\end{tabular}

Man sieht, bei einem nicht-hierarchischen Ranking haben alle Varianten gleich viele Verstöße. Ausgeschlossen wäre aber eine Form wie Czarens. Sie hätte einen ConstraintVerstoß mehr als Czare, Czaren und Czars und ist so suboptimal.

b.

\begin{tabular}{|l|l|l|l|l|l|l|l|l|l|l|}
\hline & ER- & A- & N-PL & MAX & REDFIN & N-Pl & S-PL & MARK & DEP & NO \\
STAMM- & (Cor) & SYLL & & & PL & (Mora) & Affix \\
DIFF & & & & & & & \\
\hline Czarens & & & & & $*$ & & & & $*$ & $* *$ \\
\hline
\end{tabular}




\section{$4 \quad$ Vergleich mit dem heutigen Sprachgebrauch}

Vergleicht man nun aber die behandelten Wörter mit dem heutigen Sprachgebrauch, ergeben sich andere Zuordnungen für die entsprechenden neuhochdeutschen Wörter Zobel, Möbel, Zaren, Vögte: Bei Zobel ist heute ein $n$-Plural ungrammatisch, ebensowenig kann Möbel mit einem $n$-Plural gebildet werden, und Zaren und Vögte haben keine weiteren Pluralvarianten neben sich:

c.

\begin{tabular}{|l|l|l|l|l|l|l|l|}
\hline & $\begin{array}{l}\text { MAX } \\
\text { (Cor) }\end{array}$ & $\begin{array}{l}\text { REDFIN } \\
\text { SYLL }\end{array}$ & N-Pl & S-PL [s] & MARK PL & $\begin{array}{l}\text { DEP } \\
\text { (Mora) }\end{array}$ & NO Affix \\
\hline Libertiner & & & $*$ & $*$ & $*$ & & \\
\hline Zobel & & & $*$ & $*$ & $*$ & & \\
\hline Möbel & & & $*$ & $*$ & $*$ & & \\
\hline Woiwoden & & & & $*$ & & & $*$ \\
\hline Zaren & & & & $*$ & & $*$ & $*$ \\
\hline Vögte & & & $*$ & $*$ & & $*$ & \\
\hline Taxen & & & & $*$ & & & $*$ \\
\hline Saisons & & $*$ & $*$ & & & & $*$ \\
\hline
\end{tabular}

Abgesehen davon, dass der Umlaut in Vögte festgeworden ist, hat also die Klasse der nichtfemininen Substantive, deren Endsilbe wie im Singular auf ein reduziertes sonantisches $r, l$ oder $n$ ausgeht, zugenommen. Ebenso hat sich der $n$-Plural bei den Nichtfeminina mit dem Merkmal [belebt] auf Kosten des $ə$ - oder $s$-Plurals ausgedehnt. Festgeblieben ist der $n$-Plural dagegen bei den Feminina. Ein $s$-Plural erscheint heute aber schon bei Zobel möglich, aber wohl noch nicht bei Möbel:

d.

\begin{tabular}{|l|l|l|l|l|l|l|l|}
\hline & $\begin{array}{l}\text { MAX } \\
\text { (Cor) }\end{array}$ & $\begin{array}{l}\text { REDFIN } \\
\text { SYLL }\end{array}$ & N-Pl & S-PL [s] & MARK PL & $\begin{array}{l}\text { DEP } \\
\text { (Mora) }\end{array}$ & NO Affix \\
\hline Zobels & & $*$ & $*$ & & $*$ & & $*$ \\
\hline
\end{tabular}

\section{$5 \quad$ Fazit}

Festzuhalten bleibt somit, dass das untersuchte Material bei den Fremdwörtern nicht, wie man nach der heutigen Bedeutung des $s$-Plural erwarten würde, auf einen Sprachwandel zum $s$ Affix hinweist (dazu Wegener 1999: 38; 2001: 273ff.), sondern eher auf eine klare Opposition zwischen Nichtfeminina mit einer reduzierten auslautenden Silbe einerseits und zwischen Wörtern mit $n$-Pluralen andererseits. Das sind die Maskulina mit dem Merkmal [belebt] und die Feminina. Um aber mehr über Tendenzen des morphologischen Wandels vom älteren Neuhochdeutschen zur Gegenwartssprache zu erfahren, müssten weit mehr Texte als das Schiller-Korpus ausgewertet werden.

Immerhin hat die Untersuchung des Pluralgebrauchs der Fremdwörter bei Schiller eine beträchtliche ideolektale Varianz ergeben. Diese beruht auf unterschiedlichen Faktoren: Während die nur einmal bei ihm bezeugten Formen Billeter und Veteraner wohl die hohe 
Produktivität des er-Plurals vor allem in den süddeutschen Dialekten widerspiegelt, also areal bedingt sein kann (vgl. Anm. 3), bezeugen die Pluralformen Saisons, Meubles (in französischer Aussprache) und Lustra noch keine Integration der fremdstämmigen Formen in das deutsche Flexionssystem. Anders steht es bei Vesicatorien, Woiwoden, Czaren, Taxen und Libertiner. Diese Pluralformen sind bereits der deutschen Flexion angepasst, wie sie in der Gegenwartssprache gilt. Auch Czare, Meublen und Vogte weisen einheimische Pluralendungen auf; doch haben sich diese mitsamt des fehlenden Umlauts in Vogte nicht zum Neuhochdeutschen hin durchgesetzt. Gleiches gilt für Czars, das wahrscheinlich der Flexion von Thans folgt. Schiller hat bei der Flexion der Fremdwörter also sozusagen noch "herumexperimentiert". Er war sich offenbar nicht sicher, welche Pluralendung die richtige ist.

\section{Literaturangaben}

\section{Primärliteratur}

Schillers Werke. Nationalausgabe. Begründet von Julius Peters, fortgeführt von Lieselotte Blumenthal et al. Weimar 1940ff. [noch nicht vollendet]

\section{Sekundärliteratur}

Bornschein, Matthias/Butt, Matthias (1987): "Zum Status des $s$-Plurals im gegenwärtigen Deutsch". In: Abraham, Werner/Arhammar, Rita (ed.): Linguistik in Deutschland. Tübingen: 135-153.

Duden ( $\left.{ }^{5} 1995\right)$ : Grammatik der deutschen Gegenwartssprache, hg. und bearb. von Günther Drosdowski, Mannheim (=Duden Band 4).

Eisenberg, Peter (1991): "Syllabische Struktur und Wortakzent: Prinzipien der Prosodik deutscher Wörter". Zeitschrift für Sprachwissenschaft 10: 37-64.

Grimm, Jacob/Grimm, Wilhelm (1951): Deutsches Wörterbuch; Bd. XII, Abt. II. Leipzig.

Löhken, Sylvia C. (1995): "Vowel weakening and vowel deletion in German: a constraintbased analysis". FAS Working Papers in Linguistics 2: 77-94.

Löhken, Sylvia C. (1997): Deutsche Wortprosodie. Abschwächungs- und Tilgungsvorgänge. Tübingen. (= Studien zur deutschen Grammatik 56).

Raffelsiefen, Renate (1995): "Conditions for stability: the case of schwa in German". In: Theorie des Lexikons. Arbeiten des SFB 282, Nr. 69. Heinrich-Heine-Universität Düsseldorf.

Reynolds, William T. (1994): Variation and phonological change. PhD thesis, University of Pennsylvania.

Wegener, Heide (1999): "Die Pluralbildung im Deutschen - ein Versuch im Rahmen der Optimalitätstheorie". Linguistik online 4, 3/1999.

http://www.linguistik-online.de//3_99/wegener.html

Wegener, Heide (2001): "Aufbau von markierten Pluralklassen im Deutschen - eine Herausforderung für die Markiertheitstheorie". Folia Linguistica 36: 261-295.

Wiese, Richard (1966): The phonology of German. Oxford. 
Wunderlich, Dieter (1999): German noun plural reconsidered. MS Düsseldorf.

Zubritskaya, Katya (1994): "Sound change in Optimality Theory and constraint tie". CLS 30, vol. 2: 335-349. 